\title{
Comments on “Average Life Prediction Based on Incomplete Data”
}

\author{
Tachen Liang \\ Wayne State University, Detroit, USA \\ Email: aa4156@wayne.edu
}

Received August 25, 2013; revised September 25, 2013; accepted October 6, 2013

Copyright (C) 2013 Tachen Liang. This is an open access article distributed under the Creative Commons Attribution License, which permits unrestricted use, distribution, and reproduction in any medium, provided the original work is properly cited.

\begin{abstract}
We comment on the correctness of the article “Average life prediction based on incomplete data” by [1] (Applied Mathematics, Vol. 2, pp. 93-105).
\end{abstract}

Keywords: Average Life Prediction; Censored Data

\section{Introduction}

Tang et al. [1] studied average life prediction based on incomplete data assuming the prior distribution being unknown. However, the paper contains serious errors and the concluded results are incorrect. We shall point out the errors in the following. To do so, we first describe the considered statistical model as below.

Suppose that there are $n$ different manufacture units possessing the same technology and regulations. For a known integer $m>0$, a sample of $m$ components are selected from unit $j$, and put on life test at time $t=0$, for each $j=1, \cdots, n$. It is assumed that the lifetime of a component arising from unit $j$ follows a two-parameter exponential distribution having probability density

$$
f\left(x ; \theta_{j}, \mu_{j}\right)=\frac{1}{\theta_{j}} \exp \left(\left(x-\mu_{j}\right) / \theta_{j}\right) I\left(x>\mu_{j}\right) .
$$

Let $X_{j 1}, \cdots, X_{j m}$ denote the lifetimes of the $m$ components. The life test experiment will be terminated if one of the $m$ components fails. Thus,

$X_{j}=\min \left(X_{j 1}, \cdots, X_{j m}\right)$ is the lifetime of the ineffective component from unit $j$. Let $a>0$ be a pre-specified constant. If $X_{j} \geq a$, then a second round sample is carried, at which we sample one more component from unit $j$, and denote its unknown lifetime by $Y_{j}$.

Furthermore, it is assumed that $\left(\theta_{j}, \mu_{j}\right), j=1, \cdots, n$, are iid random parameters, and that $X_{j}$ are possibly censored from the right by a non-negative random censoring variable $V_{j}$, where $V_{1}, \cdots, V_{n}$ are iid, with a common distribution $W$, and $\left(V_{1}, \cdots, V_{n}\right)$ are independent of $\left(X_{1}, \cdots, X_{n}\right)$. Thus, $X_{j}$ may not always be ob- servable. Instead, one can only observe

$Z_{j}=\min \left(X_{j}, V_{j}\right)$ and $\delta_{j}=I\left(X_{j} \leq V_{j}\right)$. Through the preceding assumptions, $\left(X_{j}, Y_{j}, V_{j}, Z_{j}, \delta_{j}, \theta_{j}, \mu_{j}\right)$,

$j=1, \cdots, n$ are iid, $\left\{V_{j}, j=1, \cdots, n\right\}$ and

$\left\{\left(X_{j}, Y_{j}, \theta_{j}, \mu_{j}\right), j=1, \cdots, n\right\}$ are mutually independent.

Let $S_{1}=\frac{1}{n} \sum_{j=1}^{n} I\left(X_{j} \geq a\right) Y_{j}$ and

$S_{2}=\frac{1}{n} \sum_{j=1}^{n} I\left(X_{j} \geq a\right) . \quad S=S_{1} / S_{2}$ is the average life of

the second round sample. Tang et al. (2011) attempted to predict $S=S_{1} / S_{2}$ based on the data

$\left(Z_{j}, \delta_{j}\right), j=1, \cdots, n$. Let

$$
\begin{aligned}
\bar{S}_{1}= & \frac{1}{n(n-1)} \sum_{i=1}^{n}\left[\sum_{j \neq i}^{n} \frac{Z_{j} \delta_{j}}{1-W\left(Z_{j}\right)}\right] \frac{I\left(Z_{i} \geq a\right) \delta_{i}}{1-W\left(Z_{i}\right)}, \\
& +\frac{(m-1)}{n} \sum_{i=1}^{n}\left(Z_{i}-a\right) \frac{I\left(Z_{i} \geq a\right) \delta_{i}}{1-W\left(Z_{i}\right)} \\
\bar{S}_{2} & =\frac{1}{n} \sum_{i=1}^{n} \frac{I\left(Z_{i} \geq a\right) \delta_{i}}{1-W\left(Z_{i}\right)}, \text { and } \bar{S}=\bar{S}_{1} / \bar{S}_{2} .
\end{aligned}
$$

Tang et al. [1] proposed using $\bar{S}$ to predict $S$. Tang et al. [1] claimed the following results:

$$
E\left[\bar{S}_{j}\right]=E\left[S_{j}\right], \quad j=1,2 .
$$

(see (2.8)-(2.9) of Tang et al. [1]).

Based on the identity property of (1), and some additional conditions, Tang et al. [1] claimed in their Theorem 1 that $\bar{S}-S \rightarrow 0$ in probability as $n \rightarrow \infty$. They 
further apply the identity property of (1) and Theorem 1 to claim their Theorem 2.

We now point out the errors of Tang et al. [1] as follows.

1) The sampling scheme is not well defined. Since random censorship model is considered, we may be unable to observe the exact value of $X_{j}$. In case that $\delta_{j}=0$ and $Z_{j}<a$, it is possible that $X_{j} \geq a$ or $X_{j}<a$. In such a situation, shall we carry the second round of sampling and sample one more component from unit $j$ ? This is not discussed in the paper.

2) For the distribution $W$ known case, unfortunately, the claimed identity that $E\left[\bar{S}_{1}\right]=E\left[S_{1}\right]$ is incorrect. The error is pointed out as follows. In (2.8) of [1], it is stated that (see Equation (2)):

In (2) (or (2.8) of Tang et al. [1]), the first equality is not true, where the notation $(\theta, \mu)$ is abused. The correct computation is given below. Note that for each $j$, given $\left(\theta_{j}, \mu_{j}\right), X_{j}$ follows a double exponential distribution having pdf $f\left(x ; \theta_{j}, \mu_{j}\right)$, and

$\left(X_{j}, V_{j}, Z_{j}, \delta_{j}, \theta_{j}, \mu_{j}\right), \quad j=1, \cdots, n$ are iid. Using the iid property, we can obtain Equation (3)

Note that the expression of (3) is different from that of (2). So, we see that $E\left[\bar{S}_{1}\right] \neq E\left[S_{1}\right]$

This type of computational errors also occur at (5.3), (5.4), (5.7), (5.8) and (5.9) of Tang et al. [1], where $E\left[S_{1}^{2}\right], E\left[S_{1} \bar{S}_{1}\right]$ and $E\left[\bar{S}_{1}^{2}\right]$ were calculated.

Based on the preceding discussion, the correctness of Theorem 1 in Tang et al. [1] is doubtful.

3) Tang et al. (2011) then applied the result of their Theorem 1 for the case where the distribution $W$ is un-

$$
\begin{aligned}
& E\left[\bar{S}_{1}\right]=\frac{1}{n(n-1)} \sum_{i=1}^{n} \sum_{j \neq i}^{n} E\left\{E\left[\frac{Z_{j} \delta_{j}}{1-W\left(Z_{j}\right)} \mid(\theta, \mu)\right] E\left[\frac{I\left(Z_{i} \geq a\right) \delta_{i}}{1-W\left(Z_{i}\right)} \mid(\theta, \mu)\right]\right\} \\
& +(m-1) E\left\{E\left[\frac{\left(Z_{1}-a\right) I\left(Z_{1} \geq a\right) \delta_{1}}{1-W\left(Z_{1}\right)} \mid(\theta, \mu)\right]\right\} \\
& =E\left\{\iint_{x \leq v} \frac{x}{1-W(x)} \mathrm{d} W(v) l(x \mid \theta, \mu) \mathrm{d} x \iint_{x \leq v} \frac{I(x \geq a)}{1-W(x)} \mathrm{d} W(v) l(x \mid \theta, \mu) \mathrm{d} x\right\} \\
& +(m-1) E\left\{\iint_{x \leq v} \frac{(x-a) I(x \geq a)}{1-W(x)} \mathrm{d} W(v) l(x \mid \theta, \mu) \mathrm{d} x\right\} \\
& =E_{(\theta, \mu)}\left\{\left(\mu+\frac{\theta}{m}\right) \exp \left(-\frac{m(a-\mu)}{\theta}\right)+\frac{(m-1) \theta}{m} \exp \left(-\frac{m(a-\mu)}{\theta}\right)\right\}=E\left[S_{1}\right] \\
& E\left[\bar{S}_{1}\right]=\frac{1}{n(n-1)} \sum_{i=1}^{n} \sum_{j \neq i}^{n} E\left\{E\left[\frac{Z_{j} \delta_{j}}{1-W\left(Z_{j}\right)} \mid\left(\theta_{j}, \mu_{j}\right)\right] E\left[\frac{I\left(Z_{i} \geq a\right) \delta_{i}}{1-W\left(Z_{i}\right)} \mid\left(\theta_{i}, \mu_{i}\right)\right]\right\} \\
& +(m-1) E\left\{E\left[\frac{\left(Z_{1}-a\right) I\left(Z_{1} \geq a\right) \delta_{1}}{1-W\left(Z_{1}\right)} \mid\left(\theta_{1}, \mu_{1}\right)\right]\right\} \\
& =E\left\{\iint_{x \leq v} \frac{x}{1-W(x)} \mathrm{d} W(v) l\left(x \mid \theta_{j}, \mu_{j}\right) \mathrm{d} x \iint_{x \leq v} \frac{I(x \geq a)}{1-W(x)} \mathrm{d} W(v) l\left(x \mid \theta_{i}, \mu_{i}\right) \mathrm{d} x\right\} \\
& +(m-1) E\left\{\iint_{x \leq v} \frac{(x-a) I(x \geq a)}{1-W(x)} \mathrm{d} W(v) l(x \mid \theta, \mu) \mathrm{d} x\right\} \\
& =E\left\{\left(\mu_{j}+\frac{\theta_{j}}{m}\right) \exp \left(-\frac{m\left(a-\mu_{i}\right)}{\theta_{i}}\right)\right\}+E_{(\theta, \mu)}\left\{\frac{(m-1) \theta}{m} \exp \left(-\frac{m(a-\mu)}{\theta}\right)\right\} \\
& =E_{\left(\theta_{j}, \mu_{j}\right)}\left\{\left(\mu_{j}+\frac{\theta_{j}}{m}\right)\right\} E_{\left(\theta_{i}, \mu_{i}\right)}\left\{\exp \left(-\frac{m\left(a-\mu_{i}\right)}{\theta_{i}}\right)\right\}+E_{(\theta, \mu)}\left\{\frac{(m-1) \theta}{m} \exp \left(-\frac{m(a-\mu)}{\theta}\right)\right\} \\
& =E_{(\theta, \mu)}\left\{\left(\mu+\frac{\theta}{m}\right)\right\} E_{(\theta, \mu)}\left\{\exp \left(-\frac{m(a-\mu)}{\theta}\right)\right\}+E_{(\theta, \mu)}\left\{\frac{(m-1) \theta}{m} \exp \left(-\frac{m(a-\mu)}{\theta}\right)\right\}
\end{aligned}
$$


known, and claimed their Theorem 2. However, since Theorem 1 is dubious, the correctness of Theorem 2 is also doubtful.

\section{REFERENCES}

[1] T. Tang, L. Z. Wang, F. E. Wu and L. C. Wang, “Average Life Prediction Based on Incomplete Data,” Applied Ma- thematics, Vol. 2, 2011, pp. 93-105.

http://dx.doi.org/10.4236/am.2011.21011 